\title{
INVESTIGATION OF THE EFFECT OF TEMPERATURE ON AGING BEHAVIOR OF Fe-DOPED LEAD ZIRCONATE TITANATE
}

\author{
NAPATPORN PROMSAWAT \\ School of Ceramic Engineering, Institute of Engineering, Suranaree University of Technology, \\ Nakhon Ratchasima 30000, Thailand \\ METHEE PROMSAWAT \\ Department of Materials Science and Technology, Faculty of Science, Prince of Songkla University, \\ Songkhla 90112, Thailand
}

PATTANAPHONG JANPHUANG

Synchrotron Light Research Institute, 111 University Avenue, Muang District, Nakhon Ratchasima 30000, Thailand

BOONRUANG MARUNGSRI

School of Electrical Engineering, Institute of Engineering, Suranaree University of Technology, NakhonRatchasima 30000, Thailand

ZHENHUA LUO

School of Water, Energy and Environment, Cranfield University, Cranfield, MK43 OAL, UK

SOODKHET POJPRAPAI*

School of Ceramic Engineering, Institute of Engineering, Suranaree University of Technology, Nakhon Ratchasima 30000, Thailand

soodkhet@sut.ac.th

Received Day Month Year;Revised Day Month Year

\begin{abstract}
The aging degradation behavior of Fe-doped PZT subjected to different heat-treated temperatures was investigated over 1000 hours. The aging degradation in the piezoelectric properties of PZT was indicated by the decrease in piezoelectric charge coefficient, electric field-induced strain, and remanent polarization. It was found that the aging degradation became more pronounced at temperature above $50 \%$ of the PZT's Curie temperature. A mathematical model based on the linear logarithmic stretched exponential function was applied to explain the aging behavior. A qualitative aging model based on polar macrodomain switchability was proposed.
\end{abstract}

Keywords: Aging; Temperature effect; Piezoelectric; PZT

Lead zirconate titanate (PZT) is a common known piezoelectric material that has excellent electro-mechanical coupling property, making the material widely studied and used for applications as sensors and actuators [1-2]. However, material's properties are weaken if the operating temperature approaches Curie temperature $\left(T_{c}\right)$, affecting the performance of the piezoelectric sensors or actuators [3-7]. Furthermore, temperature could affect aging degradation behavior of piezoelectric ceramics [8-9]. Although there are previous works reported on the aging behavior and performance of PZT actuators [10-12], temperature effect on their aging behavior is still unclear. This work aims to investigate the effects of temperature on the aging degradation of Fe-doped PZT ceramics. Fe-doped PZT was selected because of strong aging behavior. This degradation is typically sensitive to temperature. Aging behavior of the samples was quantified by mathematical model based on the stretched exponential decay function [13-16].

In our work, commercial Fe-doped PZT (PZT-4), called as hard PZT, samples with diameter and thickness of $10 \mathrm{~mm}$ and $1 \mathrm{~mm}$, respectively, were used. Chemical composition was analyzed by X-ray fluorescence spectrometer 
(XGT-5200 HORIBA Scientifile). The concentrations of Zr, $\mathrm{Ti}$ and $\mathrm{Fe}$ elements in samples were 9.00, 7.76 and 0.010 mol\%, respectively.

The $T_{C}$ of the samples is $325{ }^{\circ} \mathrm{C}$. The samples annealed at $500{ }^{\circ} \mathrm{C}$. Electrodes were deposited on the parallel sides of the samples using colloidal silver paste (Heraeus component metallization item no. OTI402). Each sample was electrically poled in a DC electric field of $3 \mathrm{kV} / \mathrm{mm}$ at $100{ }^{\circ} \mathrm{C}$ for $10 \mathrm{~min}$. Dielectric constant $\left(e_{r}\right)$, Piezoelectric constant $\left(d_{33}\right)$, strainelectric field $(S-E)$ loop and polarization-electric field $(P-E)$ hysteresis loop after poling were measured via a LCR-meter (GW Instek LCR-821), a d33-meter (APC products inc. S5865), a strain sensor in conjunction with a high voltage supplier (Trek, model 20/20C) and a Sawyer-Tower circuit, respectively. Poled samples were subjected to heat treatment at different temperatures $\left(30,40,50,60,70\right.$ and $80 \%$ of the $\left.T_{C}\right)$ with a dwell time of $10 \mathrm{~min} .24 \mathrm{hr}$ after heat treatment, aging behavior in $e_{r}, d_{33}, S-E$ and $P-E$ loops was measured over a period of $1,000 \mathrm{hr}$. The value of $e_{r}$ before heat treatment did not change with aging time. However, $e_{r}$ significantly decreased with the heat treatments at temperatures below $0.8 T_{C}$ while $e_{r}$ suddenly increased with the treatment at $0.8 T_{C}$. This abrupt increase in $e_{r}$ could be caused by a decrease in interaction between poled domains due to the applied thermal energy. After heat treatment, $e_{r}$ gradually degraded with increasing aging time. This was due to the clamping dipole moments of defect dipoles [17]. The heat treatment temperatures did not have significant effect on the aging rate of the samples. The $d_{33}$ values measured before and after heat treatment were normalized to the value measured at the beginning of aging process. Time dependence of normalized $d_{33}$ of samples heated at different temperatures is shown in Fig. 1. The $d_{33}$ measured before heat treatment gradually degraded with aging time. This is due to a relaxation effect of piezoelectric domains after poling. The slow decay reflects the rearrangement of domains towards equilibrium states. The driving force for this rearrangement could be come from elastic or electric i.e. relaxation of the internal stress or internal field opposing the polarization, respectively [16]. After heat treatment, the aging degradation become more significant due to the strong thermal agitation on poled domains. The domains become easier to switch since the heat treatment lowered the interaction strength between macrodomains and thus their switching activation energy [18-19]. This is further supported by the X-ray diffraction (XRD) pattern obtained from the samples before and after heat treatment, as shown in Fig. 2, measured by an XRD instrument (D2 PHASER, Bruker, USA). The rhombohedral and tetragonal phases of the (200) peaks are

Fig. 1. Time dependence of normalized ${ }_{d 33}$ of PZT ceramics after heat treatment at different temperatures. The solid lines are fitting curves obtained

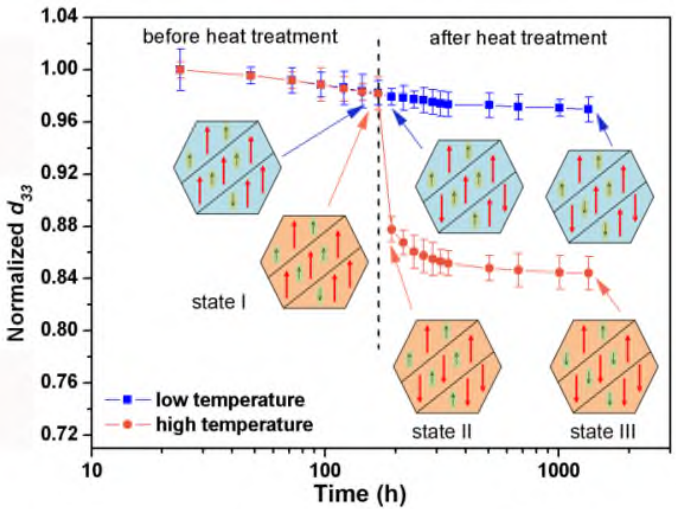

from the equations (1). The schematic model showed the orientations of dipole moments of spontaneous polarizations (red long arrows) and defect dipoles (green short arrows) in a grain (represented by an octagon) during aging processes before and after heat treatments at lower and higher temperatures.

represented by subscript $\mathrm{R}$ and $\mathrm{T}$, respectively. The intensity of (200)R peak increases after the sample is thermally treated. The ratio of the intensities of (002) $\mathrm{T}$ and (200)R peaks are calculated. These ratio values before poling, after poling, 1 day and 35 days after heat treatment are equal to $0.92,1.44,1.04$ and 1.11 , respectively. This indicates that the poling process could orient the majority of the unit cells in the [002]T direction, which is parallel to the applied electric field direction. After the heat treatment, some unit cells reorient to original $(200)_{\mathrm{R}}$ directions. However, the $(200)_{\mathrm{T}}^{\mathrm{T}}$ phase does not recover after the heat treatment. It also shows that the aging time had less significant effect than the heat treatment.

At the beginning of the heat treatment, the domains that initially aligned in the poling direction by poling are reversed. This results in the decrease of $d_{33}$ with an increase in temperature, as shown in Fig.1. To quantify the aging behavior of the PZT, the time dependent degradation is represented by the change of $d_{33}$ value as a function of time. Time dependent degradation of normalized $d_{33}$ after heat treatment is fitted to the stretched exponential function, as the following equation $[10,11]$

$$
d=d \square+d_{1} \exp [-(t / v)]
$$

where $d$ is the normalized value constant. $d x$ is the time independence part. $d_{1}$ is the part of the sample constant varying with time. $t, z^{-}$and $v$ are the aging time, the characteristic relaxation time and a stretching exponent, respectively. Fig. 1 shows the result of the normalized $d_{33}$ as 
a function of time after the heat treatment at 30 to $80 \%$ of Curie temperature, respectively. The fitting curves obtained equation (1) is displayed as solid lines. The fitting parameters from data in Fig. 1 are listed in Table 1. The z- value reflects the aging rate and $v$ is the fitting parameter of the linear logarithmic function. Based on the equation (1), $v$ varies in the range from 0.1 to 1 . It shows that the calculated results from equation (1) is consistent to the experimental values. Equation (1) also shows that $z$ - decreases when the ratio of $\left(d_{1} / d_{\infty}\right)$ increases as the temperature $t$ increases. This indicates an increase in the aging rate as a function of the heat treatment temperature. This is also consistent with the experimental result in Fig. 1.

The results in Fig. 1 could be explained by a schematic model. It presents that the orientations of spontaneous polarizations and defect dipoles embedded in a ceramic's grain. It is generally known that spontaneous polarizations are formed below Curie's temperature due to non-centrosymmetry of structures. Besides the spontaneous polarization, defect dipoles could be formed due to a creation of oxygen vacancies. Their dipole moments can be reoriented by high applied fields. After the high field is removed, defect dipoles gradually arrange their directions anti-parallel to directions of neighboring spontaneous polarizations until reaches the equilibrium state. There are three observation states: I) after poling and before heat treatment, II) just after heat treatment and III) after the aging processes. The orientations of the polarization and dipoles are represented by the directions of the red and green arrows, respectively. The grains of the samples subjected to low and high heat treatment temperatures are shown as blue and pink grains, respectively. In the grains, the domains are separated by domain walls. In this model, the reorientation of defect dipole is taken into account for describing the aging degradation of the ceramics properties [19]. Before the heat depolarized, the

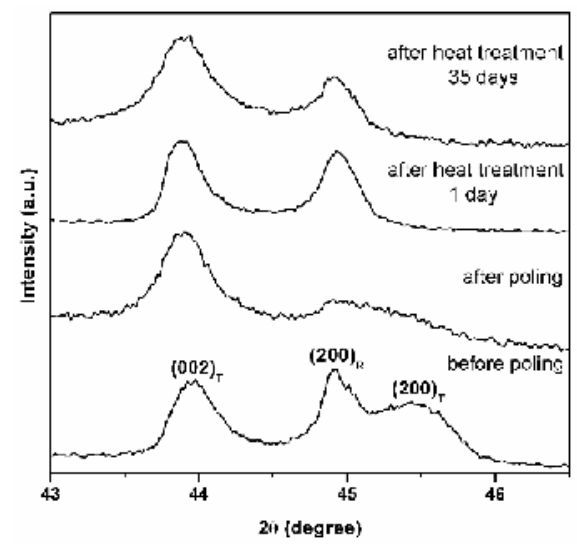

Fig. 2. The XRD patterns at 20 ranged from $43-46.5^{\circ}$ at difference states of the hard PZT ceramics. number of misaligned arrows in the grains treated at high temperature (pink) are compared to the ones treated at lower temperature (blue). Furthermore, in Fig.3, as the aging time increases and thermal energy is no longer supplied to the system, the grains heated treated at higher temperature degrades faster than the one treated at lower temperature. This is caused by the reorientation of defect dipoles. As proposed by Sapper et al., [20] naturally, defect dipole usually oriented their dipole moment in the direction of the polar macrodomains, at which those defects are embedded. At the aging process of the samples which are treated at higher temperature, more defect dipoles are reoriented since more de-poled polarizations are generated during the heat treatment process, leading to a further decrease in the degree of poling compared to those samples treated at a lower temperature, as shown in State III in Fig. 1. Therefore, higher aging rate is observed in the samples that are heat treated at higher temperature. Aging behavior of $S-E$ and $P-E$ loops is shown in Fig. 3. The values of strain and remanent polarization $\left(P_{r}\right)$ abruptly decrease with heat treatment temperature. The degradation of strain and $P_{r}$ increases with increasing heat treatment temperature. Interestingly, the samples treated at $0.3 T_{C}$ and $0.5 T_{C}$ show stronger degree of pinched loop compared to $0.8 T_{C}$ sample. This might be caused by an effect of the de-clamping domains of defect dipoles due to thermal energy at higher temperature near $T_{C}$. In conclusion, the aging degradation in $d_{33}, e_{r}, S-E$ and $P-E$ loops of hard PZT ceramics are investigated at different heat treatment temperatures. Weak aging in the properties is observed at the heat treatment temperature less than $50 \%$ of $T_{\mathrm{c}}$. On the contrary, the aging degradation becomes more severe when the temperature excesses $50 \%$ of $T_{\mathrm{c}}$. This could be because macropolarizations are depolarized at heat treatment, where the number of depoled macrodomains increased with increasing temperature, leading to a stronger degradation of properties. Furthermore, an increase in number of depoled macrodomains drives the reorientation of defect dipoles. Thus, when the samples are treated at higher temperature, more defect dipoles are reoriented in the sample even after the heat treatment, leading to a more severe aging degradation. The effect of heat treatment temperature on the aging behavior could be explained by a mathematical model

Table 1. Fitting parameters of normalized $d_{33}$ of PZT ceramics after heat treatment at different temperatures obtained from the equation (2).

\begin{tabular}{|c|c|c|c|c|c|c|}
\hline Fitting & \multicolumn{6}{|c|}{ Heat treatment temperature } \\
\hline$d_{l} / d_{\infty}$ & 0.0112 & 0.0153 & 0.0373 & 0.0476 & 0.1029 & 0.1624 \\
\hline z- (hr) & 114.35 & 89.81 & 69.98 & 38.94 & 21.13 & 7.01 \\
\hline v & 0.6809 & 0.4985 & 0.2979 & 0.2990 & 0.2550 & 0.2840 \\
\hline
\end{tabular}



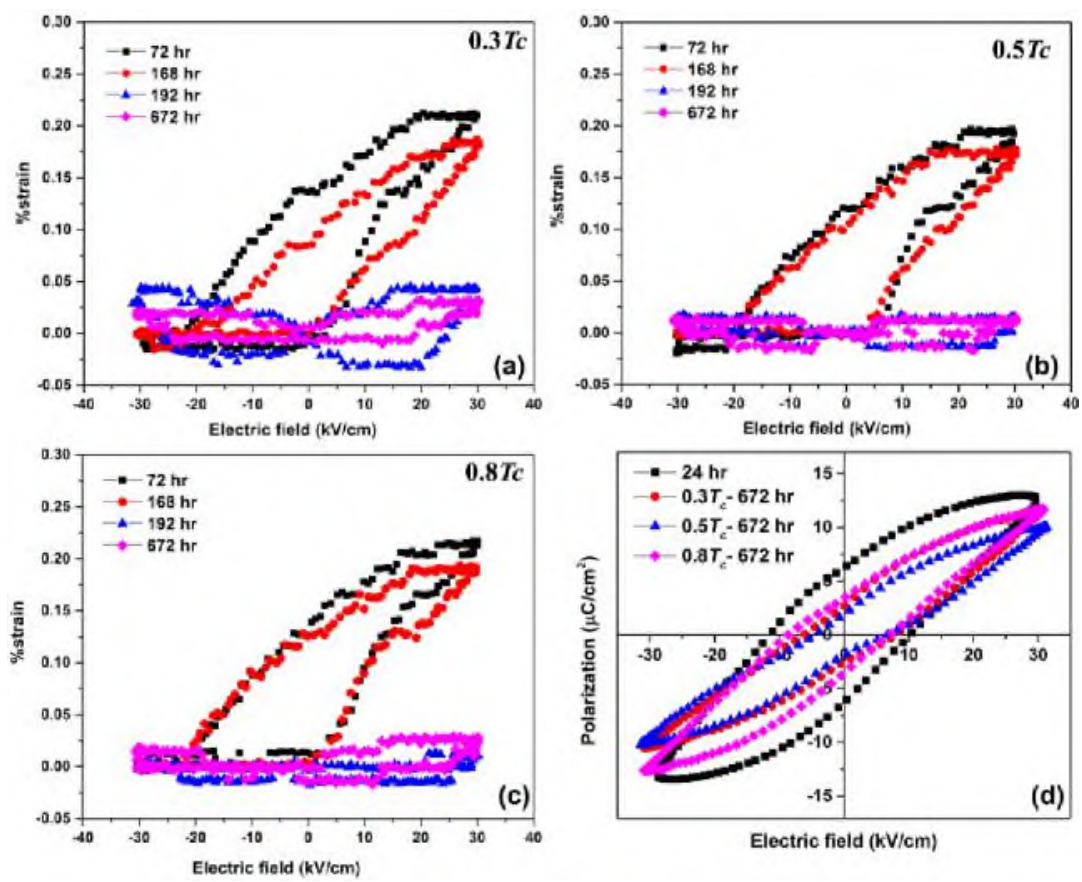

Fig. 3. The $S-E$ loops at different time after heat treatments at (a) $0.3 T c$, (b) $0.5 T_{c}$ and (c) $0.8 T c$. (d) The $P$-E hysteresis loops measured at different aging times of hard PZT.

using linear logarithmic stretched treatment, the poled ceramics, in which the polarizations of polar macrodomains and defect dipoles oriented in the direction of the poling field, are slightly aged as in State I. At the heat treatment, the poled macrodomains become depolarized due to the thermal agitation. This model could be used to predict the aging degradation in PZT and could be useful to optimizing the thermal treatment process in applications.

\section{Acknowledgments}

This research has been supported by Suranaree University of Technology and the Higher Education Research Promotion and National Research University Project of Thailand, Office of the Higher Education Commission. The authors thank A. Phuetthonglang for his preliminary data and guidance.

\section{References}

1. J. Rödel et al., J. Eur. Ceram. Soc. 35, 1659 (2015).

2. K. G. Webber et al., J. Am. Ceram. Soc. 97, 2842 (2014).

3. Y. A. Genenko et al., Mater. Sci. Eng., B. 192, 52 (2015).

4. M. I. Morozov et al., J. Appl. Phys. 107, 034106 (2010).

5. G. Xu et al., Appl. Phys. Lett. 86, 032902 (2005).

6. D. A. DeAngelis et al., IEEE Ultrason. Symp. 1935 (2006).

7. M. Alguero et al., J. Eur. Ceram. Soc. 20, 2705 (2000).

8. A. Barzegar et al., J. Appl. Phys. 89, 2322 (2001).

9. S. Su et al., Curr. Appl Phys. 11, S120 (2011).

10. J. F. Shepard et al., J. Appl. Phys. 85, 6711 (1999).

11. H. Dederichs et al., Ferroelectrics. 68, 281 (1986).

12. A. L. Kholkin et al., J. Electroceram. 19, 81 (2007
13. Q. M. Zhang et al., J. Appl. Phys. 79, 3181 (1996).

14. J. H. Koh et al., J. Appl. Phys. 96, 544 (2004).

15. S. Priya et al., App. Phys. Lett. 79, 2624 (2001).

16. D. Xue et al., Appl. Phys. Lett. 94, 082902 (2009).

17. U. Robels et al., Ferroelectrics. 168, 301 (1995).

18. W. Pan et al., J. Am. Ceram. Soc. 75, 1534 (1992).

19. C. Brennan, Ferroelectrics. 150, 199 (1993).

20. E. Sapper et al., J. Appl. Phys. 116, 104102 (2014). 